\title{
Inteligencia emocional y su relación en el desempeño laboral
}

\section{Emotional intelligence and its relationship in the job performance}

\section{Como citar el artículo}

Aragón, J., (2019). Inteligencia emocional y su relación en el desempeño laboral. Revista Naturaleza, Sociedad y Ambiente, 6 (1),55-68 DOI: https://doi.org/10.37533/cunsurori.v6i1.41

\section{Kehilly Izabel Aragón Zepeda}

Psicóloga Clínica, Maestra en Gerencia Administrativa de Recursos Humanos

Recibido: 27 de septiembre de 2019 / Aceptado: 04 de noviembre de 2019

Disponible en internet el 29 de noviembre de 2019

*Autor para correspondencia, correo electrónico: kehilly@gmail.com

\begin{abstract}
Resumen
Se conoce que el desempeño laboral se refiere al cumplimiento exitoso de tareas asignadas, eficacia y eficiencia en cada una de las funciones que exige su cargo. El desempeño laboral dentro de las organizaciones tiene muchos factores relacionados con la personalidad de los colaboradores. Actualmente el departamento de recursos humanos toma en cuenta la personalidad de cada empleado, como sus actitudes, cualidades, formas de pensar y comportarse. Los factores psicológicos y emocionales son de gran importancia al momento de evaluar el desempeño de los mismos. La inteligencia emocional es la capacidad humana de sentir, entender, controlar y modificar estados emocionales en sí mismo y en los demás, posee componentes clave para afrontar los diferentes desafíos en el campo laboral, como la motivación que ayuda a tomar la iniciativa y aprovechar las oportunidades, la capacidad de relacionarse con los demás e influir, trabajar y comunicarse con otras personas, la empatía que implica reconocer y leer emociones en los demás. Una persona empática es sensible hacia otras personas, las comprende y fomenta el desarrollo de las mismas, la autoconciencia que hace referencia a tener conocimiento de sí mismo, autorregulación que es la capacidad de manejar y controlar el propio estado emocional.
\end{abstract}

Palabras clave: inteligencia emocional, desempeño laboral, perfil del líder emocional, componentes de la inteligencia, aplicación de la inteligencia emocional

\begin{abstract}
It is known that work performance refers to the successful fulfillment of assigned tasks, effectiveness and efficiency in each of the functions required by their position. Work performance within organizations has many factors related to the personality of employees. Currently the human resources department takes into account the personality of each employee, such as their attitudes, qualities, ways of thinking and behaving. The psychological and emotional factors are of great importance when evaluating their performance. Emotional intelligence is the human capacity to feel, understand, control and modify emotional states in oneself and in others, it has key components to face the different challenges in the labor field, such as the motivation that helps to take the initiative and take advantage of the opportunities, the ability to interact with others and influence, work and communicate with other people, the empathy that involves recognizing and reading emotions in others. An empathetic person is sensitive to other people, understands them and encourages their development, the self-consciousness that refers to having knowledge of oneself, self-regulation that is the ability to manage and control one's emotional state.
\end{abstract}

Keywords: emotional intelligence,work performance, profile of the emotional leader, components of intelligence, application of emotional intelligence 


\section{Introducción}

La inteligencia emocional es muy importante en el desarrollo organizacional, ya que cuenta con componentes que son indicadores para afrontar los diferentes desafíos en la empresa. La inteligencia emocional tiene factores que ayudan al buen desempeño laboral, como motivación, habilidades sociales, auto-regulación, autoconciencia, seguridad, empatía, etc. Actualmente se ha evidenciado la falta de estos factores en muchas empresas o instituciones. Por ejemplo la falta de motivación, falta de autocontrol y manejo de emociones, falta de empatía y otros diversos factores que están relacionados con la Inteligencia Emocional que juegan un papel en el buen desempeño laboral. Hacer este tipo de investigación ayudará a muchas instituciones a través de las recomendaciones a diagnosticar los distintos o posibles factores que pueden afectar la eficiencia y eficacia de sus colaboradores en sus labores.

El departamento de recursos humanos tiene una gran responsabilidad y a la vez la oportunidad de afrontar nuevos retos, ya que han de mantener un contacto continuo con las personas que conforman la empresa o institución, tanto en el momento en que realizan la entrevista de selección como a la hora de gestionar el desarrollo de los trabajadores. Sin embargo, han surgido nuevas formas de trabajar y ello implica nuevos retos a los que han de enfrentarse actualmente, como aprender a reconocer e identificar factores que pueden estar relacionados con el buen desempeño de los colaboradores. El orientar esta investigación hacia el lado psicológico es porque se tenía como objetivo principal conocer y determinar si la inteligencia emocional está relacionada o influye en el buen desempeño laboral.

\section{Referente teórico}

\section{Inteligencia emocional}

Goleman (2004), define el término inteligencia emocional como la capacidad humana de sentir, entender, controlar y modificar estados emocionales en uno mismo y en los demás. Por lo que relacionando esta definición tenemos que tomar en cuenta lo que refería Chiavenato (2002), el trato que se les vaya a dar a los colaboradores, la manera de integrarlas, motivarlas, mantenerlas y orientarlas es un factor clave para el alcance y éxito de objetivos fijados dentro de la organización.

Ríos (2015), afirma que la inteligencia emocional es un conjunto de destrezas, actitudes, habilidades y competencias que determinarán la conducta de un individuo, sus reacciones y estados mentales. Además la inteligencia emocional se compone de distintos factores cómo lo es la auto regulación, motivación, empatía y las relaciones interpersonales y que esta determina cómo las personas se manejan a sí mismas y a con los demás. Sostiene que existen una serie de habilidades que se pueden aprender basadas en sentimientos y emociones, las cuales determinan el éxito o el buen desempeño de las tareas.

La inteligencia emocional es el poder de automotivación, capacidad de manejar emociones internas y relaciones externas. Una persona que posee inteligencia emocional es capaz de buscar alternativas para tener una vida plena y feliz, aprenden cada día y son muy firmes en sus decisiones, tienen buenas relaciones con los demás, aprovecha sus cualidades y afronta las adversidades, sobretodo tienen muchos beneficios y ventajas porque controlan sus propias emociones y tienen auto-motivación y autocontrol. Refiere que las 
emociones y sentimientos han ganado importancia en todos los ámbitos de vida (Acuña, 2017).

Riveros (2017), refiere que desde la época de los filósofos griegos se ha identificado que las emociones constituyen una característica de los seres humanos. Además refiere que existen diversas teorías que amparan el concepto de la inteligencia emocional, por ejemplo, cita a Salovey y Mayer (1990), definieron la inteligencia emocional como la capacidad que tiene un individuo de identificar los sentimientos propios y ajenos. Así mismo cita a Goleman (1995), la inteligencia emocional es la habilidad de identificar los sentimientos y las emociones propias y las de los demás, y de gestionar las relaciones con otros. Es decir, es un conjunto de destrezas, actitudes, habilidades y competencias que determinan la conducta de un individuo, sus reacciones, estado mental, es una capacidad potencial que determina el aprendizaje de habilidades prácticas basadas en una de las siguientes características o componentes de la inteligencia emocional: como la conciencia de uno mismo, la motivación, el autocontrol, la empatía y la capacidad de relación.

Maturrano (2015), refiere que las personas exitosas no son necesariamente aquellas que obtuvieron las mejores calificaciones en la escuela, la capacidad intelectual no es pronóstico o resultado de un óptimo desempeño laboral, para ello es necesario tomar en cuenta o demostrar otras competencias personales que son características propias de la inteligencia emocional.

McClelland (1989), planteó que las actitudes académicas tradicionales como las calificaciones y los títulos no nos permiten predecir el grado de desempeño laboral que tendrá un colaborador en una empresa o el éxito que éste tendrá en la vida. Proponía que los rasgos que diferencias a los trabajadores sobresalientes de aquellos otros que simplemente hacen bien las cosas había que buscarlos en competencias tales como la empatía, la autodisciplina y la motivación.

Goleman (2001), indica ciertas habilidades que posee una persona con inteligencia emocional:

- Conciencia de sí mismo y de las propias emociones y su expresión

- Autorregulación

- Control de impulsos

- Control de la ansiedad

- Diferir las gratificaciones

- Regulación de estados de ánimo

- Motivación

- Optimismo ante las frustraciones

- Empatía

- Confianza en los demás

- Artes sociales 
Elementos

$\begin{array}{lll} & & \\ \text { Suto-regulación } & \checkmark \\ & \text { y controlar el propio } & \checkmark \\ & \text { estado emocional. } & \\ & & \checkmark \\ & \\ & \\ & \\ & \\ & & \checkmark \\ & \text { Conocerse a sí mismo } & \checkmark \\ & \text { y saber lo que dicen } & \checkmark \\ & \text { las emociones. } & \checkmark\end{array}$

Autoconciencia

Motivación

Canalizar emociones para alcanzar metas.

\section{Aspectos por aplicar}

Posponer juicios; refrenar impulsos.

Dejar el problema; alejarse de este.

Expresarse, pero de manera asertiva, no agresiva.

Ser flexible; no forzar las cosas.

Manejar la comunicación no verbal.

\section{Respetarse.}

Ser Positivo.

Ser fiel a sí mismo.

Dar un descanso a la lógica y a la racionalidad.

Escuchar a los demás.

Entender el impacto en los demás.

Luchar por mejorar y alcanzar estándares

altos.

Comprometerse a alcanzar sus metas.

Tomar la iniciativa y aprovechar las oportunidades.

Ser optimista incluso en presencia de la adversidad.

Ser sensible hacia otras personas y comprenderlas.

Convertir las necesidades e intereses de otros en puntos de referencia.

Fomentar el desarrollo de otras personas. Estar en sintonía con lo social y lo político.

Desarrollar y mantener relaciones interpersonales.

Habilidades

Relacionarse con los $\checkmark$ Comunicarse con los demás. Sociales demás e influir en $\checkmark$ ellos.

Tabla 1 Componentes de la inteligencia emocional

Fuente:Valenica y Salazar (2012). 


\section{Desempeño laboral}

Chiavenato (2002), indica que el rendimiento laboral se refiere a la capacidad que tiene una persona de producir, hacer, elaborar, acabar y generar trabajo con mejor calidad en menos tiempo y con menor esfuerzo, se define como la eficacia o capacidad del personal que se evalúa lo cual dará como resultado su desenvolvimiento dentro de la organización.

Pereira (2012), el desempeño laboral es el grado en el que el empleado cumple los requisitos de su puesto de trabajo, además afirma que la evaluación de desempeño es un proceso de reducción de incertidumbre y al mismo tiempo de búsqueda de consonancia. No obstante, Stoner (1994:510), afirma "el desempeño laboral es la manera como los miembros de la organización trabajan eficazmente, para alcanzar metas comunes, sujeto a las reglas básicas establecidas con anterioridad". El desempeño laboral se refiere a la ejecución de las funciones por parte de los empleados de una organización de manera eficiente, con la finalidad de alcanzar las metas propuestas.

Goleman (1999), indica que el concepto de inteligencia emocional aplicado al trabajo, demuestra que quienes alcanzan altos niveles dentro de las organizaciones poseen un gran control de sus emociones, están motivadas y son generadoras de entusiasmo. Saben trabajar en equipo, tienen iniciativa y logran influir en los estados de ánimo de sus compañeros. Aptitudes como el autoconocimiento, la seguridad en uno mismo, y el autocontrol, el compromiso, la integridad y la habilidad de comunicarse con eficacia son algunas de las características que analizó con lucidez e intuición, por ende, demostró que estas capacidades son las requeridas en el mercado de trabajo actual. También afirmaba que el éxito profesional y persona está más ligado a las competencias emocionales que al $\mathrm{Cl}$, y que entre un $70 \%$ y un $90 \%$ del éxito depende de competencias emocionales y el resto de aptitudes intelectuales.

Dessler (2002), hace mención que el departamento de recursos humanos toma en cuenta la personalidad de cada empleado, debido a que todos tienen diferentes actitudes, cualidades, formas de pensar y comportarse; al poseer un cargo en recursos humanos, son factores que se deben analizar y tener presente. Uno de los retos que tiene el encargado de recursos humanos es la innovación y creatividad. Sin embargo, es importante contar con un encargado profesional en recursos humanos con un amplio conocimiento a lo que refiere reclutamiento, selección, capacitación, motivación y mantenimiento, aparte de las funciones básicas administrativas necesarias; contar y aplicar los pasos del proceso administrativo. Además otro de los desafíos es adquirir empleados comprometidos y satisfechos con su trabajo. Muchas de las dificultades que se presentan en este departamento son el crecimiento y desarrollo del empelado fuera del espacio y tiempo laboral, se debe estimular la motivación y bienestar del empleado, realizando actividades o convivencias sociales en familia y entorno del individuo, etc.

Olvera et al. (2002), mencionan que el éxito dentro de una organización depende de muchos factores más que solo de la capacidad intelectual o técnica, que para sobrevivir y superarse en el mercado laboral se requiere de dominio y otras habilidades. Entre los cuales se pueden mencionar la actitud del colaborador, la personalidad, es decir, su temperamento y carácter, el pensamiento muy importante la manera cómo se percibe e interpreta, 
las diferentes emociones que se presentan ante determinada situación, el ambiente laboral, físico, social, personal y clima laboral.

\section{Perfil del líder emocional}

En la actualidad se conocen ciertas cualidades que posee un líder emocional. Se toman como base cinco ejes dentro de este perfil:

- Liderazgo: se refiere a la conceptualización, autoconfianza, uso de presentaciones orales.

- Gestión de los recursos humanos: manejo de procesos de grupo, uso de poder socializado.

- Énfasis en los demás: objetividad perceptual, autocontrol, resistencia y adaptabilidad.

- Objetivos y acción: se refiere a la preocupación por el impacto, uso diagnóstico de los conceptos y orientación en la eficacia.

- Conocimiento especializado

Tomando en cuenta estas cualidades de un líder emocional es importante definir el liderazgo inteligente en las organizaciones y cómo resultado es necesario responder algunas interrogantes:

- ¿Cómo provoca el líder emocional, la emoción de los clientes para que se entusiasmen con los productos y servicios de la empresa?

- ¿Cómo inspirar a los colaboradores para que se comprometan con un objetivo compartido?

- ¿Cómo podría un líder sin autoconocimiento o autoconciencia rendir al $100 \%$ en su trabajo?

- ¿Cómo sería un líder que no controla su nerviosismo o su ira en el momento de una reunión comercial con un cliente?

- ¿Cómo un líder sin motivación podrá cen- trarse en realizar un trabajo con ilusión, entregar una sonrisa sincera y una conversación alegre o generar ideas creativas?

- ¿Cómo un líder sin empatía puede descubrir con facilidad si un cliente se siente inseguro, nervioso o si desconfía de algo?

- ¿Cómo un líder sin habilidades de relaciones interpersonales, podría comunicarse con sus colaboradores o clientes?

Aplicación de los componentes de la inteligencia emocional por parte de un líder

- Conocimiento de uno mismo: el lídersabe reconocer que sentimientos afectan el trabajo, existe conciencia emocional de sí mismo, confianza y una valoración adecuada de sí mismo.

- Asertividad: el líder sabe expresar los sentimientos de forma sincera y respetando, a la vez, las posturas de los demás.

- Autocontrol: el lídercanaliza los sentimientos negativos fuera del trabajo, existe transparencia, adaptabilidad, logro, iniciativa, optimismo y autogestión.

- Empatía: el lídercomprende a los demás aunque muestren puntos de vista diferentes, hay conciencia social, servicio y conciencia organizativa.

- Perseverancia: un líder no puede tirar la toalla, debe motivar siempre al equipo.

- Motivación: el líder tiene deseos por conseguir logros que aporten un enorgullecimiento personal y para la empresa.

- Gestión de las relaciones: el líder cataliza el cambio, maneja la gestión de los conflictos, trabaja en equipo y colaboración, implementa el desarrollo personal de los demás, hay influencia e inspiración. 


\section{Metodología}

Se empleó el estudio de casos, se utilizaron pruebas estadísticas aplicando el programa Excel, utilizando cantidades porcentuales para el análisis crítico. También se utilizó la observación ya que permite conocer la realidad mediante la percepción directa de los fenómenos alrededor. La técnica que se utilizó fue una encuesta para determinar si existe relación o influye la inteligencia emocional con el desempeño laboral.

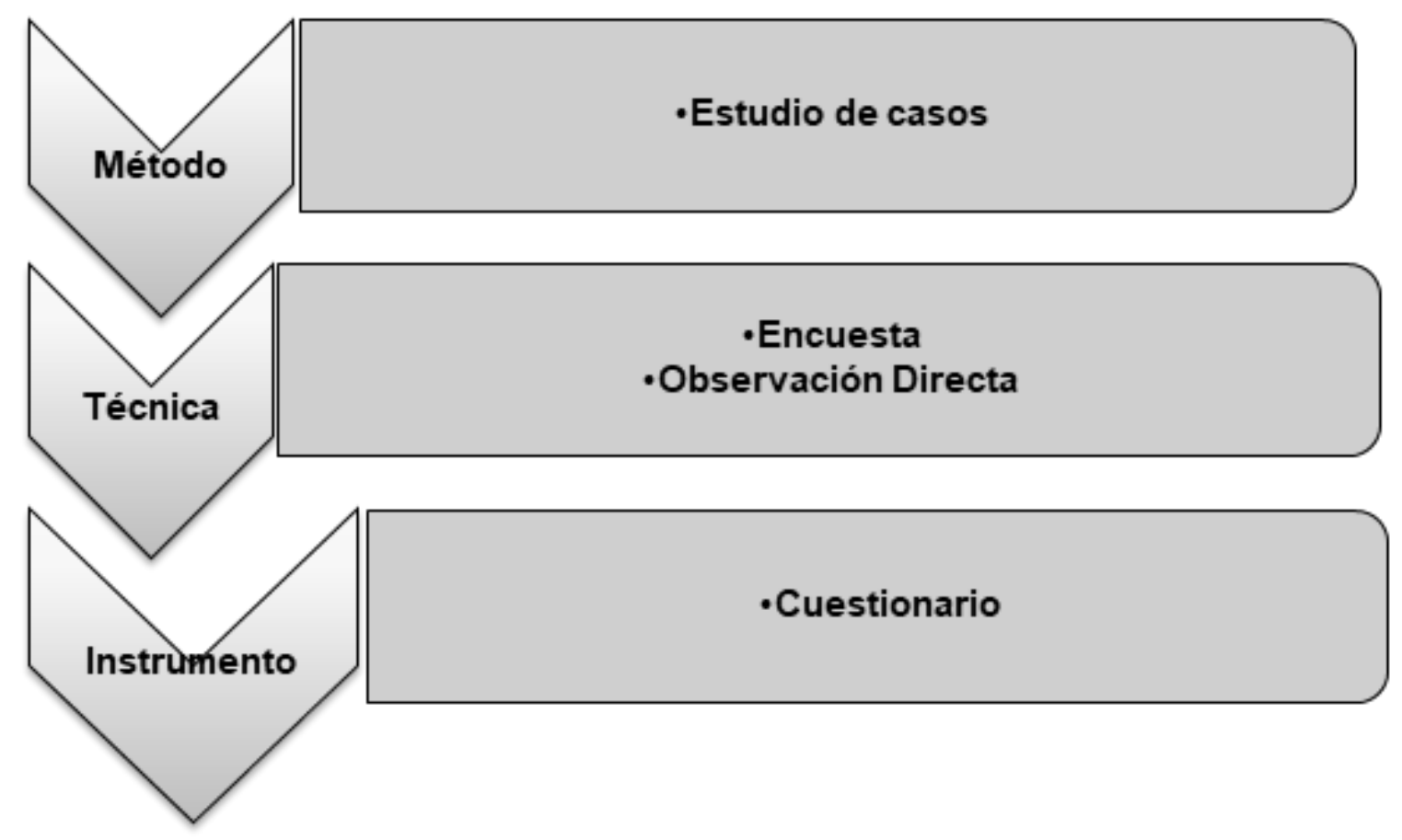

Figura 1

\section{Resultados}

Para realizar esta investigación se tomaron como base cinco preguntas que responden a los resultados finales que se obtuvieron:

1. ¿Cómo se encuentra la capacidad de manejar y controlar el propio estado emocional de los colaboradores?

2. ¿Los colaboradores reconocen y fomentan el desarrollo de otras personas?
3. ¿Los colaboradores muestran optimismo y luchan por mejorar y alcanzar estándares altos?

4. ¿Incide la autoconciencia en el desempeño laboral de los colaboradores?

5. ¿Existen buenas relaciones interpersonales y trabajo en equipo en los colaboradores? 


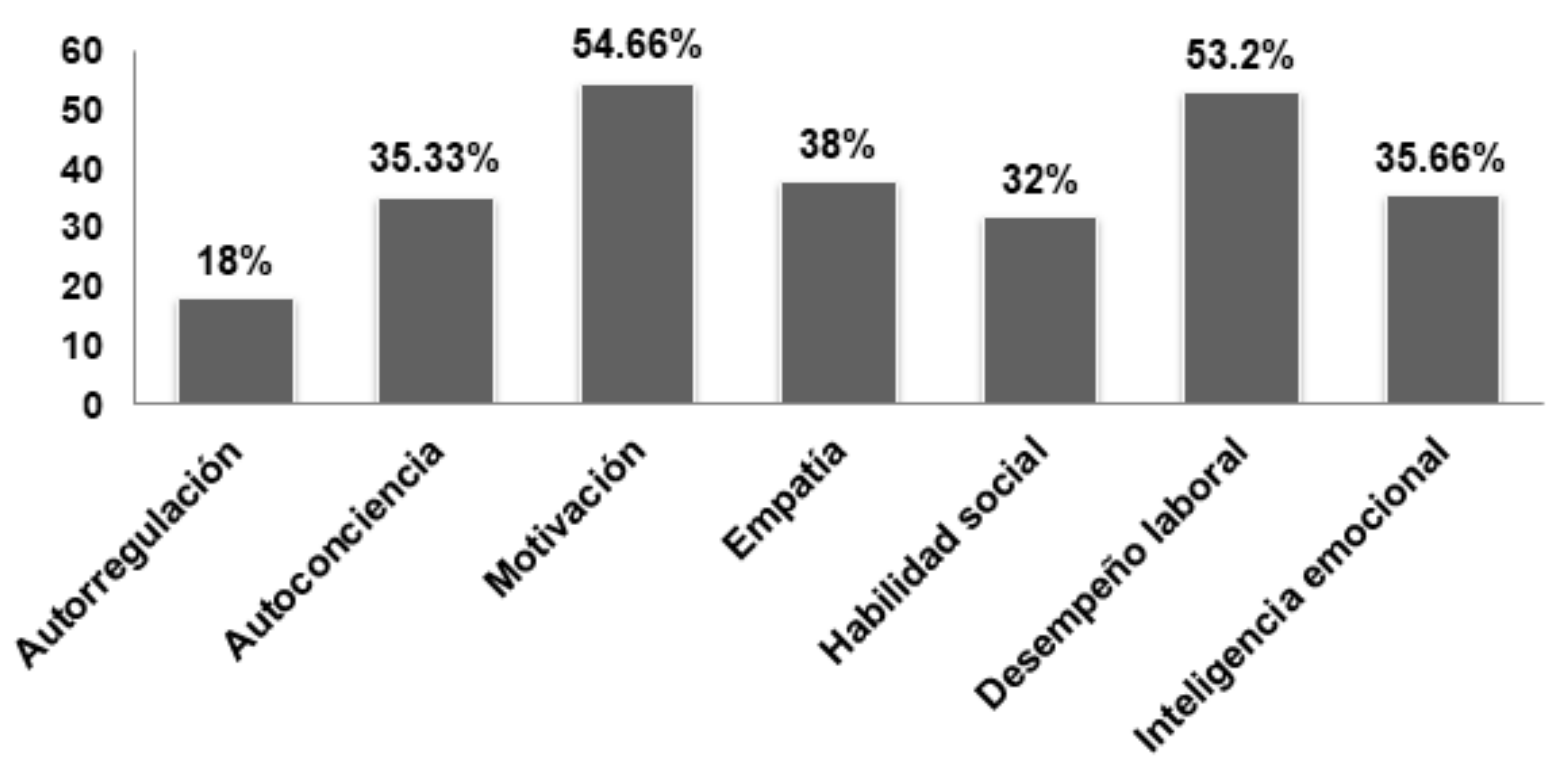

Gráfica 1 Inteligencia emocional y su relación en el desempeño laboral

Fuente: Elaboración propia

Goleman (2004), refiere que la inteligencia emocional juega un papel importante en el desempeño laboral dentro de una empresa. Según resultados obtenidos se puede observar que los colaboradores encuestados manejan cada uno de los componentes de la inteligencia emocional. En estudio el $54.66 \%$ están motivados en su área de trabajo ya que están dispuestos a trabajar, tienen esa actitud que se necesita a diario dentro de una organización. Un trabajador feliz, es un trabajador motivado y optimista, un trabajador feliz desarrolla todo su talento y da mucho más de sí mismo. (Rojas, 2010) .
Londoño (2008), la autorregulación dentro de una empresa es muy importante ya que se mantiene el control de nuestro estado anímico, impulsos y recursos internos y en esta investigación el $18 \%$ poseen autorregulación. Un $35.33 \%$ autoconciencia y el $38.33 \%$ posee empatía.

López (2007), las relaciones interpersonales autenticas se basan en el reconocimiento de la categoría humana de toda persona, a través del trato con respeto y servicio. Mostrarlas y sentirlas con el equipo de trabajo contribuye a mejorar la relación y amistad, se puede analizar que un $32 \%$ de la población estudiada tiene habilidades sociales. 


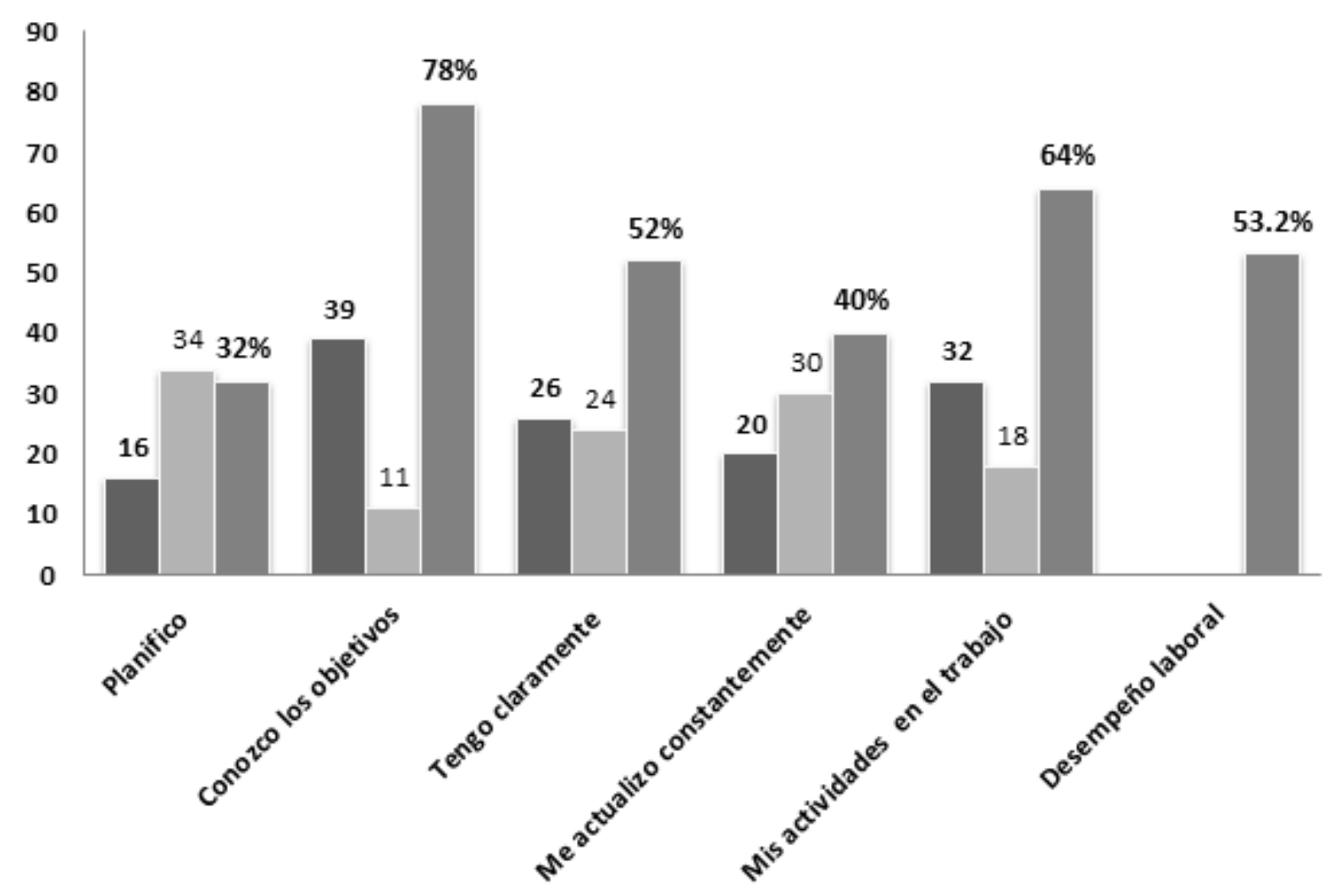

Gráfica 2 Desempeño laboral

Goleman (1999), indica que el concepto de inteligencia emocional aplicado al trabajo, demuestra que quienes alcanzan altos niveles dentro de las organizaciones poseen un gran control de sus emociones, están motivadas y son generadoras de entusiasmo. Saben trabajar en equipo, tienen iniciativa y logran influir en los estados de ánimo de sus compañeros. Respecto al desempeño el $53.2 \%$ tiene un alto desempeño laboral, se actualizan constantemente, realizan sus tareas enfocadas en sus funciones específicas y con eficiencia. Trabajan arduamente a través de objetivos y metas. Conocen la visión y misión de la institución. Además planifican sus actividades de acuerdo a los requisitos de su puesto de trabajo. Se obtuvo en promedio un $35.66 \%$ de los porcentajes de cada uno de los componentes de la inteligencia emocional, se puede analizar que si se relaciona con el desempeño laboral.
McClelland (1989), refería que era importante brindar reconocimientos a los colaboradores por su buen desempeño, por lo que es necesario que las empresas tengan y mantengan este manejo de incentivos que impacta positivamente en los colaboradores.

\section{Conclusiones}

La aplicación de los distintos elementos o áreas de la inteligencia emocional en el campo laboral, permite mejorar notablemente con eficacia el desempeño laboral. La aplicación y el manejo de la inteligencia emocional en el trabajo brindan grandes beneficios no sólo para los colaboradores sino también para las empresas o instituciones, se obtendría un excelente equipo de colaboradores con grandes aptitudes y habilidades, las cuales se pondrían en práctica en el campo laboral, 
como se puede analizar en esta investigación el $53.2 \%$ ejerce sus funciones con eficiencia, conoce cuáles son sus objetivos, trabajan en equipo, existe una plena satisfacción laboral, lo que observa en el porcentaje obtenido en los ejes evaluados del desempeño laboral.

El $35.66 \%$ de la población tomada para la investigación maneja la inteligencia emocional y por ende tiene una influencia o relación en el desempeño. Es decir, si existe una adecuada motivación ya que muestran optimismo y luchan por mejorar y alcanzar estándares altos, auto-regulación ya que tiene la capacidad de manejary controlar el propio estado emocional, autoconciencia, empatía porque reconocen y fomentan el desarrollo de otras personas y tienen excelentes interpersonales y fomentan el trabajo en equipo dentro de la institución lo que se evidencia en el desempeño laboral.

\section{Referencias}

Acuña, E. (2017). Inteligencia Emocional y su Desempeño Laboral. Perú.

Chiavenato, I. (2002). Gestión del Talento Humano. 3ra. Edición. Editorial Mc-Graw Hill. España

Chiavenato, I. (2002), Administración de recursos humanos. $5^{\mathrm{a}}$. Edición. Editorial Mc Graw Hill. Colombia

Dessler, G. (2002). Administración de Recursos Humanos. 5a. Edición. Editorial Pearson Educación México.

Goleman, D. (1998). Working with Emotional Intelligence. New York: Bantam Books (Traducción al castellano, Kairós, 1999).

Goleman, D. (2004). La inteligencia emocional en la empresa. Argentina: Vergara.

Goleman, D. (1995). Emotionalintelligence.
New York: Bantam Books (Traducción Castellano, Kairós 1999).

Llano, A. (2015). La Inteligencia Emocional en Puestos Directivos. Universidad Autónoma de Ciudad Juárez. España.

Londoño, M. (2009). Como sobrevivir al cambio: inteligencia emocional y social en la empresa. FC Editorial. Madrid.

López, T. (2007). Humanizar las Relaciones Interpersonales. Colombia.

Maturrano, T. (2015). Inteligencia emocional y su relación con el desempeño laboral de los docentes del nivel primario.

Mayer, D. y P. Salovey. (1997). ¿What is emotional intelligence? Emotional Development and Emotional Intelligence: Implications for Educators. Pp.3-31. New York.

McClelland, D. (1989). Estudio de la Motivación Humana. Ediciones Narcea. España.

Olvera, Y., B. Domínguez, y A. Cruz. (2002). Inteligencia emocional, Manual para profesionales en el ámbito industrial. Primera Edición. Editorial Plaza y Valdés, S.A. México.

Pereira, S. (2012). Nivel de inteligencia emocional y su influencia en el desempeño laboral. Universidad Rafael Landivar. Guatemala.

Ríos, T. (2015). "Inteligencia Emocional y su relación con el Desempeño Laboral". Pascana. Pág. 4.

Riveros, A. (2017). La inteligencia emocional en el desempeño laboral de docentes de instituciones de educación superior. Universidad Autónoma de México. México.

Rojas, P. (2010). "La Felicidad en el trabajo sinónimo de productividad" Senior Manager. 
Salazar, J. (2012). La inteligencia emocional Valencia, G. (2012). Inteligencia Emocional en en el éxito empresarial. Editorial Universitaria Abya Yala. Ecuador. el Éxito Empresarial. Editorial Universitaria Abya Yala. Quito Ecuador.

Salovey, P., y Mayer, J. (1990). Emotional intelligence. Imagination, Cognition and Personality, 9, P.p. 185-211.

\section{Sobre autora}

\section{Kehilly Izabel Aragón Zepeda}

Estudió 1er y 2do año de Médico y Cirujano en Universidad de San Carlos de Guatemala, Centro Universitario de Oriente CUNORI. Universidad Mariano Gálvez, Extensión Jalapa, Licenciatura en Psicología Clínica. Diplomado en Logoterapia y Análisis Existencial en el Instituto de Ciencias de la Familia Guatemala, Asociación Guatemalteca de Logoterapia. Maestría en Gerencia Administrativa de Recursos Humanos, Universidad de San Carlos de Guatemala Centro Universitario CONSURORI.

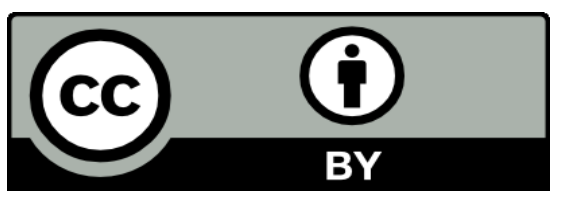

Este texto está protegido por una licencia CreativeCommons 4.0.

Esta licencia permite que otros distribuyan, mezclen, adapten y desarrollen su trabajo, incluso comercialmente, siempre y cuando le den crédito por la creación original. 